\title{
Uncoordinated Cooperative Forwarding in Vehicular Networks with Random Transmission Range
}

\author{
Xuefei Zhang*, Guoqiang Mao ${ }^{\dagger \ddagger *}$, Xiaofeng Tao* and Qimei Cui* \\ * Beijing University of Posts and Telecommunications, China \\ $\dagger$ University of Technology Sydney, Australia $\quad \ddagger$ National ICT Australia (NICTA), Australia
}

\begin{abstract}
This paper investigates cooperative forwarding in large highly dynamic vehicular networks. Unlike traditional coordinated cooperative forwarding schemes that require a large amount of coordination information to be exchanged before making the forwarding decision, this paper proposes an uncoordinated cooperative forwarding scheme where each node, with a random transmission range, decides whether or not to forward a received packet independently based on a forwarding probability determined by its own location. Analytical results are derived on the successful end-to-end transmission probability and the expected number of forwarding nodes involved in the cooperative forwarding process. The multi-hop correlations and multi-path correlations, which constitute major challenges in the analysis, are carefully considered in our analysis. Simulations are conducted to establish the performance of the proposed scheme assuming different forwarding probability functions. In addition to developing an uncoordinated cooperative forwarding scheme, which is particularly suited for the highly dynamic vehicular networks, this paper also makes important theoretical contributions on analyzing the connectivity of networks with nodes of variable and random transmission ranges.
\end{abstract}

\section{INTRODUCTION}

This paper considers the problem of cooperative forwarding in highly dynamic vehicular networks. To measure the performance of multi-hop cooperative forwarding schemes, two important metrics are often used: the end-to-end transmission success probability and the number of forwarding nodes. Of course, if every node overhearing the packet between a source and a destination forwards the packet with a high probability, the packet can be delivered to its destination with a high end-to-end transmission success probability but it may cause a large number of redundant transmissions thereby wasting precious radio resources. On the other hand, if the forwarding probability is low which reduces the number of forwarding nodes, the packet may not eventually arrive at its destination.

End-to-end packet transmissions in vehicular networks can be either in single hop, i.e. transmissions only occur when the source and the destination are within the transmission range of each other, or via multiple hops. There are two common approaches to end-to-end packet transmissions: broadcast and cooperative communication. Broadcast remains to be the most reliable and possibly the most widely used approach for packet transmission $[1,2]$ however it is well known to cause a large number of redundant transmissions and significant waste of radio resources. Cooperative communication on the other hand allows additional nodes in the vicinity of the route that overhear the transmitted packet to selectively assist in delivering the packet to its destination, leveraging the broadcast nature of the wireless communication to provide the diversity gain [3]. An optimally designed cooperative communication strategy may reduce the number of forwarding nodes involved in the transmission while guaranteeing a pre-designated transmission success probability.

A common feature in existing cooperative techniques is the coordination required among the participating neighbors. These coordinations typically include the discovery of neighbors in the vicinity, the collection of channel information to these neighbors, and the selection of the best neighbor(s) whose cooperation will maximize the performance [1, 3-6]. It was reported in [7] that the coordination overhead may account for 99 percent of the total packet transmissions. The heavy overhead involved in coordinating transmissions of forwarding nodes may offset the benefits of cooperative transmissions and negatively impact the performance of endto-end transmissions, particularly in highly dynamic vehicular networks. Due to the associated coordination overheads, existing cooperative communication methods are suitable mostly for static networks and cannot work well in highly dynamic networks. As we have started to see the large-scale deployment of vehicular networks, it is imperative to develop the cooperative communication method for highly dynamic vehicular networks.

Node transmission range is a key factor in determining the connectivity of highly dynamic networks. A dynamic network is said to be connected if there is a path from any node to any other node in the network. Obviously, connectivity is a prerequisite for end-to-end transmissions to be successful. Most of existing research on connectivity assume that each node has an identical and fixed transmission range, which may not be appropriate for real networks considering the complex radio propagation environment and the heterogeneity in wireless devices. Comparatively, the random transmission range model, where each node has a random transmission range drawn from a certain range, better reflects reality in wireless networks because it can account for variability in the communication links and the radio environment. For example, in vehicular networks a node close to a high-rise building in CBD may have a smaller transmission range compared with a node in an open space on a highway.

Motivated by the above observations, in this paper we consider an uncoordinated cooperative forwarding scheme for vehicular networks, where each node with a variable 
and random transmission range overhearing a packet makes forwarding decisions independently without prior coordination with or measurement of channel information to its neighbors, and even without being aware of their existence. Related work on the uncoordinated cooperative forwarding schemes include $[8,9]$, which considered cooperative transmission schemes employing respectively a constant forwarding probability [8], a forwarding probability which is a linear function of the distance between the transmitter and the receiver [9], or a forwarding probability determined jointly by the distance to the destination and the spatial distribution of nodes [1]. In [3], theoretical analysis was presented on the successful transmission probability using three uncoordinated forwarding schemes in two-hop scenario where the source and the destination are at most two hops away. Further, reference [4] investigated the optimal forwarding scheme in the two-hop scenario. In [10], the analytical result on optimal uncoordinated cooperative forwarding probability in multi-hop scenarios was derived. But the authors ignored the correlation between multiple paths and multiple hops along the same path between the source and the destination by modeling the transmission process as a branching process so as to obtain an upper bound on the successful transmission probability. The work in [11] analyzed the connectivity of 1-D wireless networks considering a finite number of nodes randomly deployed in a given region where each node has a random communication range by utilizing existing results on the circle covering problem [12]. The authors however have incorrectly ignored some subtle but important differences between the circle covering and connectivity of 1D network caused by the boundary effects. Furthermore the analytical result on successful transmission probability is also expressed in a complicated form.

In this paper, we aim to fill an important theoretical gap left by previous work on 1D network connectivity and on uncoordinated cooperative forwarding schemes by deriving the exact analytical result on the successful transmission probability and the expected number of cooperative transmissions involved in end-to-end communications considering the correlations between multiple paths and multiple hops along the same path between the source and the destination. More specifically, the main contributions of this paper are:

- Considering one-dimensional highly dynamic networks where each node has a random transmission range, this paper proposes an uncoordinated cooperative forwarding scheme, where each node receiving the packet makes forwarding decisions independently of other nodes, using its own location, without prior coordination with its neighbors and even without being aware of their existence.

- The exact analytical results on the successful end-toend transmission probability and the expected number of forwarding nodes involved in the multihop uncoordinated cooperative communication are obtained, considering the correlation between multiple paths and multiple hops between the source and the destination.
- Developing an optimum uncoordinated cooperative forwarding scheme, which is particularly suited for the highly dynamic vehicular networks

- Simulations and discussions are presented to validate the performance of the proposed uncoordinated cooperative forwarding scheme under different forwarding probability functions.

\section{Problem Formulation}

In this paper, we consider a vehicular network on a highway. The vehicular network is modeled as a one-dimensional (1D) network on a linear segment of length $L$ with a single source-destination pair. Without loss of generality, we assume that the source is located at the origin and the destination is located on $+x$ axis. The probability distribution function of the distance between a pair of randomly chosen nodes in a 1-D vehicular network can be readily obtained. Therefore, it is straightforward to extend the results obtained in this paper to 1-D networks with multiple source-destination pairs.

A total of $n$ nodes, apart from the source at $x_{0}=0$ and the destination at $L$, are randomly and uniformly distributed within $(0, L)$. Let $x_{1}, x_{2}, \ldots, x_{n}$ be the locations of these nodes and each node has a random transmission range drawn identically, independently and uniformly from $\left[R_{\min }, R_{\max }\right]$. Let $r_{i}$ be the transmission range of the $i-t h$ node: $r_{i} \sim U\left(R_{\min }, R_{\max }\right)$. A node is in either an active mode or a silent mode. A node is in the active mode if the node can receive or forward an arriving packet; otherwise the node is in the silence mode. Initially, all nodes are in the active mode and the transition from the active mode to the silent mode will be described later. Fig. 1 illustrates an instance of a network where the triangle is the source, the square is the destination, circle nodes are the nodes, and lines with arrow denote the corresponding transmission ranges. As commonly done in the literature [1, 3, $4,7]$, when we consider the transmission of a packet between a source and a destination, movement of nodes during the endto-end transmission is not considered. That is, we consider a snap-shot of the vehicular network at a particular time instant. A typical end-to-end transmission can be completed in the order of milliseconds, during which the movement of nodes (vehicles) is comparatively small.

Moreover, we assume that each node knows its own location, which can be readily obtained from GPS or via one of the numerous wireless localization techniques available, the location of the destination, which can be carried in the packet header. Using the location information, the node makes forwarding decision independently without prior coordination with its neighbors and even without being aware of their existence. More specifically, after the source broadcasts a packet, the following rule is used in making a forwarding decision when a node located at $x \in(0, L)$ receives a packet:

- If the node receives a copy of the packet for the first time, it calculates the forwarding probability using its own location, and decides whether to forward the packet according to the forwarding probability $p(x)$. The forwarding probability function is a design parameter 


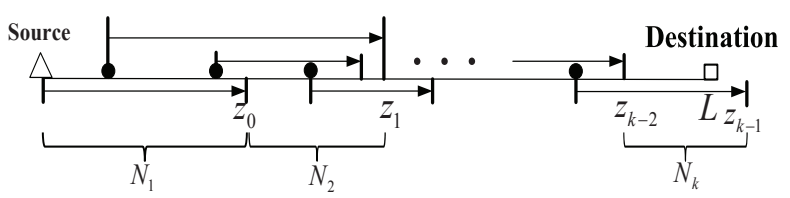

Fig. 1. An illustration of the farthest $k$-hop transmitter.

that will be used to optimize the performance of the uncoordinated forwarding scheme.

- If the node decides to forward the packet, it will transmit the packet and then switch to the silent mode. Otherwise, it drops the packet and then switches to the silent mode.

- The transmission naturally stops when there is no node forwarding in the network.

Note that in our forwarding scheme, (1) it is assumed that a proper MAC mechanism (i.e. CSMA) is employed to avoid collision; and (2) a node forwards at most once.

A node at $x$ is said to be a $k$-hop receiver if when the packet is received by the node for the first time, the packet has been transmitted $k$ times by nodes located within $[0, x)$ including the transmission by the source. A node at $x$ is said to be a $k$-hop transmitter if the node is a $k$-hop receiver and decides to transmit the received packet. Now, we give another important definition: the endpoint location of a $k$ hop transmitter, denoted by $y_{k}$, is equal to the location of the node location $x_{k}$ plus its transmission range $r_{k}$ of the node, i.e. $y_{k}=x_{k}+r_{k}$. A node at $x$ is said to be the farthest $k$-hop transmitter if the node is a $k$-hop transmitter and its endpoint location is nearest to the destination among all $k$ hop transmitters. We denote by $z_{k}$ the endpoint location of the farthest $k$-hop transmitter. Following the definition, the source is both the 0-hop transmitter and also the farthest 0hop transmitter, and $z_{0}$ is equal to the transmission range of the source. Let $N_{k}$ be the number of $k$-hop transmitters. Fig. 1 illustrates relationship of the terms defined above.

A transmission from the source is said to be successful if at least one copy of the packet successfully reaches the destination at $L$. Let $M(L)$ be the random number of forwarding nodes which have participated in transmitting the packet but may not necessarily result in a successful end-to-end transmission to the destination at $L$. Our objective is to evaluate the successful end-to-end transmission probability, denoted by $P_{\text {suc }}(L)$, and the expected number of the forwarding nodes, denoted by $E[M(L)]$. An optimally designed cooperative transmission scheme should minimize $E[M(L)]$ subject to the constraint that $P_{\text {suc }}(L)$ is above a certain designated threshold.

\section{Problem Analysis}

In this section, we first derive the analytical result on the successful transmission probability $P_{\text {suc }}(L)$ and the expected number of forwarding nodes $E[M(L)]$ for a general case. Then, An optimally designed cooperative transmission scheme is provided.

The successful end-to-end transmission probability can be expressed as

$$
P_{\text {suc }}(L)=\sum_{m=0}^{n} P_{\text {suc }}(L \mid m) \operatorname{Pr}(Q=m)
$$

where $P_{\text {suc }}(L \mid m)$ is the successful transmission probability conditioned on having $m$ relay nodes and $\operatorname{Pr}(Q=m)$ is the probability that the random number of relay nodes $Q$ is equal to a given integer $m$. For convenience, we make a distinction between "forwarding nodes", denoted by $M(L)$, and the "relay nodes", denoted by $Q$. We count those nodes who will forward, if they receive the packet, as "relay nodes". On the other hand, all nodes, who have transmitted the packet, are counted as "forwarding nodes" no matter whether the endto-end transmission is successful or not.

The expected number of forwarding nodes can be expressed as

$$
E[M(L)]=\sum_{m=0}^{n} E[M(L) \mid m] \operatorname{Pr}(Q=m)
$$

where $E[M(L) \mid m]$ is the expected number of forwarding nodes conditioned on having $m$ relay nodes.

Due to the independence of the forwarding decision of each node, the probability of having $m$ relay nodes is given by:

$$
\operatorname{Pr}(Q=m)=\left(\begin{array}{c}
n \\
m
\end{array}\right) \beta^{m}(1-\beta)^{n-m}
$$

where $\beta$ is the probability that a randomly chosen node is a relay node:

$$
\beta=\frac{1}{L} \int_{0}^{L} p(x) d x
$$

and $p(x)$ is the forwarding probability of the node at $x$, i.e. the probability that the node at $x$ decides to forward the packet, if the node receives the packet.

The joint probability density function (pdf) of locations of $m$ relay nodes and the number of relay nodes $Q=m$ for $m \geq 1$ can be expressed as

$$
\begin{aligned}
& g\left(x_{1}, \ldots, x_{m}, x_{m+1}, \ldots, x_{n}, Q=m\right) \\
= & \left(\begin{array}{c}
n \\
m
\end{array}\right) \prod_{i=1}^{m} f_{X}\left(x_{i}\right) p\left(x_{i}\right) \prod_{j=m+1}^{n} f_{X}\left(x_{j}\right)\left[1-p\left(x_{j}\right)\right]
\end{aligned}
$$

where $f_{X}(x)$ is the pdf of the location of a randomly chosen node (out of all $n$ nodes). Therefore, we can easily obtain the pdf of the location of a relay node at $x$, denoted by $g_{X}(x)$, conditioned on the number of relay nodes $m$ for $m \geq 1$ using the total probability theorem and the Baye's formula.

$$
g_{X}(x \mid Q=m)=\frac{1}{\beta} f_{X}(x) p(x)=g_{X}(x)
$$

From the first equality in (6), we can see that the conditioned pdf $g_{X}(x \mid Q=m)$ is independent of the number of relay nodes $m$.

Recall that $z_{k}$ is the endpoint location of the farthest $k$-hop transmitter, whose pdf is denoted by $h\left(z_{k}\right)$. The successful 
transmission probability from the source to the destination conditioned on having $m$ relay nodes can be expressed as

$$
\begin{aligned}
P_{\text {suc }}(L \mid m)= & \operatorname{Pr}\left(z_{0} \geq L \mid m\right)+ \\
& \sum_{k=2}^{K_{m}} \operatorname{Pr}\left(z_{k-1} \geq L, z_{k-2}<L \mid m\right)
\end{aligned}
$$

where $K_{m}$ is the maximum number of hops between the source and the destination and can be set to $n$ in the computation. $\operatorname{Pr}\left(z_{0} \geq L \mid m\right)=\operatorname{Pr}\left(z_{0} \geq L\right)=$ $\int_{\max \left(L, R_{\min }\right)}^{R_{\max }} f_{R}\left(r_{0}\right) d r_{0}$ is the probability that the destination is a 1-hop receiver, and $f_{R}(r)$ is the pdf of the transmission range of a relay node.

For $k \geq 2$, the probability that the destination is a $k$ hop receiver conditioned on having $m$ relay nodes can be calculated as

$$
\begin{gathered}
\operatorname{Pr}\left(z_{k-1} \geq L, z_{k-2}<L \mid m\right)=\sum_{m_{1}, m_{2}, \ldots, m_{k-1} \in \Gamma} \\
\int_{R_{\min }}^{R_{\max }} \int_{z_{0}}^{L} \ldots \int_{z_{k-3}}^{L} \int_{L}^{z_{k-2}+R_{\max }} \\
\xi\left(z_{k-1}, \ldots, z_{0}, N_{k-1}=m_{k-1}, \ldots, N_{1}=m_{1} \mid m\right) \\
d z_{k-1} \ldots d z_{0}
\end{gathered}
$$

where $\Gamma=\left\{m_{1}, m_{2}, \ldots, m_{k-1}: m_{1}, m_{2}, \ldots, m_{k-1}>\right.$ 0 and $\left.m_{1}+m_{2}+\ldots+m_{k-1}=m\right\}, N_{k}$ is the random number of $k$-hop transmitters, $m_{i}$ is a positive integer, $\xi\left(z_{k-1}, \ldots, z_{0}, N_{k-1}=m_{k-1}, \ldots, N_{1}=m_{1} \mid m\right)$ is the joint pdf of the endpoint locations and numbers of 0-hop, 1-hop,..., $(k-1)$-hop transmitters conditioned on having $m$ relay nodes.

Note that a relay node at $x$ is the farthest $k$-hop transmitter if and only if the following conditions are met:

- The node is a $k$-hop receiver. That means the node is located within $\left(z_{k-2}, z_{k-1}\right)$.

- The endpoint of the node is closest to the destination among all $k$-hop transmitters.

Therefore, using the chain rule in probability theory and noting that 1) $z_{k-1}$ is only dependent on $z_{k-2}, z_{k-3}$ and $m_{k-1}$ and 2) $m_{k-1}$ is dependent on $z_{k-2}, z_{k-3}, m_{1}, m_{2}, \ldots, m_{k-2}$ and $m$, the joint pdf can be calculated as

$$
\begin{aligned}
& \xi\left(z_{k-1}, \ldots, z_{0}, m_{k-1}, \ldots, m_{1} \mid m\right) \\
= & h\left(z_{k-1} \mid z_{k-2}, z_{k-3}, m_{k-1}\right) \\
& \operatorname{Pr}\left(m_{k-1} \mid z_{k-2}, z_{k-3}, m_{1}, m_{2}, \ldots, m_{k-2}, m\right) \\
& \ldots h\left(z_{1} \mid z_{0}, 0, m_{1}\right) \operatorname{Pr}\left(m_{1} \mid z_{0}, 0, m\right) f_{R}\left(z_{0}\right)
\end{aligned}
$$

where $h\left(z_{k-1} \mid z_{k-2}, z_{k-3}, m_{k-1}\right)$ is the pdf of $z_{k-1}$ conditioned on $z_{k-2}, z_{k-3}$ and $N_{k-1}=m_{k-1}, f_{R}\left(z_{0}\right)$ is the pdf of the transmission range and $\operatorname{Pr}\left(m_{k-1}\right.$ $\left.z_{k-2}, z_{k-3}, m_{1}, m_{2}, \ldots, m_{k-2}, m\right)$ is the probability that the number of $(k-1)$-hop transmitters is $m_{k-1}$ conditioned on $z_{k-3}, z_{k-2}, N_{1}=m_{1}, N_{2}=m_{2}, \ldots, N_{k-2}=m_{k-2}$ and $Q=m$.

Now, we calculate $\operatorname{Pr}\left(m_{k-1} \quad \mid \quad z_{k-2}, z_{k-3}, m_{1}, m_{2}\right.$, $\left.\ldots, m_{k-2}, m\right)$. For $k \geq 2$, it can be shown using Baye's formula and that $N_{k-1}$ has a binomial distribution condition on $z_{k-3}, z_{k-2}, N_{1}=m_{1}, N_{2}=m_{2}, \ldots, N_{k-2}=m_{k-2}$ and $Q=m$ that

$$
\begin{gathered}
\operatorname{Pr}\left(N_{k-1}=m_{k-1} \mid z_{k-3}, z_{k-2}, m_{1}, m_{2}, \ldots, m_{k-2}, m\right)= \\
\left(\begin{array}{c}
m-\sum_{i=1}^{k-2} m_{i} \\
m_{k-1}
\end{array}\right)\left[G_{X}\left(z_{k-2}\right)-G_{X}\left(z_{k-3}\right)\right]^{m_{k-1}} \\
\frac{\left[G_{X}(L)-G_{X}\left(z_{k-2}\right)\right]^{m-\sum_{i=1}^{k-1} m_{i}}}{\left[G_{X}(L)-G_{X}\left(z_{k-3}\right)\right]^{m-\sum_{i=1}^{k-2} m_{i}}}
\end{gathered}
$$

where $G_{X}(x)$ is the cumulative distribution function (cdf) of a relay node's location, and $G_{X}(b)-G_{X}(a)=\int_{a}^{b} g_{X}(x) d x$ is the probability that a relay node is located within $a$ and $b$. Similarly, the probability that the number of 1-hop transmitters equals to $m_{1}$ conditioned on $z_{0}$ can be expressed as

$$
\begin{gathered}
\operatorname{Pr}\left(N_{1}=m_{1} \mid 0, z_{0}, m\right)=\left(\begin{array}{c}
m \\
m_{1}
\end{array}\right)\left[G_{X}\left(z_{0}\right)-G_{X}(0)\right]^{m_{1}} \\
{\left[G_{X}(L)-G_{X}\left(z_{0}\right)\right]^{m-m_{1}}}
\end{gathered}
$$

Then, in order to obtain $h\left(z_{k-1} \mid z_{k-2}, z_{k-3}, m_{k-1}\right)$, we need to calculate the conditional pdf (conditional on $Q=m$ ) of the endpoint location of $(k-1)$-hop transmitter, denoted by $\eta_{Y}\left(y_{k-1} \mid z_{k-3}, z_{k-2}\right)$. Recall that $y_{k-1}$ is the endpoint location of a $(k-1)$-hop transmitter, i.e. $y_{k-1}=x_{k-1}+r_{k-1}$. Since the two random variables $x_{k-1}$ and $r_{k-1}$ are independent and further noting that $x_{k-1}$ is within $\left[z_{k-3}, z_{k-2}\right]$, according to the pdf of sum of two independent random variable, $\eta_{Y}\left(y_{k-1} \mid z_{k-3}, z_{k-2}\right)$ can be expressed as

For $k=1$, if $z_{0}+R_{\min }<R_{\max }$

$$
\begin{aligned}
& \eta_{Y}\left(y_{1} \mid 0, z_{0}\right)= \\
& \left\{\begin{array}{c}
\frac{G\left(y_{1}-R_{\min }\right)}{G\left(z_{0}\right)\left(R_{\max }-R_{\min }\right)}, \\
R_{\min } \leq y_{1} \leq z_{0}+R_{\min } \\
\frac{1}{R_{\max }-R_{\min }}, \\
z_{0}+R_{\min } \leq y_{1} \leq R_{\max } \\
\frac{G\left(z_{0}\right)-G\left(y_{1}-R_{\max }\right)}{G\left(z_{0}\right)\left(R_{\max }-R_{\min }\right)}, \\
R_{\max } \leq y_{1} \leq z_{0}+R_{\max }
\end{array}\right.
\end{aligned}
$$

Otherwise,

$$
\begin{gathered}
\eta_{Y}\left(y_{1} \mid 0, z_{0}\right)= \\
\left\{\begin{array}{c}
\frac{G\left(y_{1}-R_{\min }\right)}{G\left(z_{0}\right)\left(R_{\max }-R_{\min }\right)}, \\
R_{\min } \leq y_{1} \leq R_{\max } \\
\frac{G\left(y_{1}-R_{\min }\right)-G\left(y_{1}-R_{\max }\right)}{G\left(z_{0}\right)\left(R_{\max }-R_{\min }\right)}, \\
R_{\max } \leq y_{1} \leq z_{0}+R_{\min } \\
\frac{G\left(z_{0}\right)-G\left(y_{1}-R_{\max }\right)}{G\left(z_{0}\right)\left(R_{\max }-R_{\min }\right)},, \\
z_{0}+R_{\min } \leq y_{1} \leq z_{0}+R_{\max }
\end{array}\right.
\end{gathered}
$$


For $k>2$, if $z_{k-2}-z_{k-3}<R_{\max }-R_{\min }$,

$$
\begin{gathered}
\eta_{Y}\left(y_{k-1} \mid z_{k-3}, z_{k-2}\right)= \\
\left\{\begin{array}{c}
\frac{G\left(y_{k-1}-R_{\min }\right)-G\left(z_{k-3}\right)}{\left(G\left(z_{k-2}\right)-G\left(z_{k-3}\right)\right)\left(R_{\max }-R_{\min }\right)}, \\
z_{k-3}+R_{\min } \leq y_{k-1}<z_{k-2}+R_{\min } \\
\frac{1}{R_{\max }-R_{\min }}, \\
z_{k-2}+R_{\min } \leq y_{k-1}<z_{k-3}+R_{\max } \\
\frac{G\left(z_{k-2}\right)-G\left(y_{k-1}-R_{\max }\right)}{\left(G\left(z_{k-2}\right)-G\left(z_{k-3}\right)\right)\left(R_{\max }-R_{\min }\right)}, \\
z_{k-3}+R_{\max } \leq y_{k-1}<z_{k-2}+R_{\max }
\end{array}\right.
\end{gathered}
$$

Otherwise,

$$
\begin{gathered}
\eta_{Y}\left(y_{k-1} \mid z_{k-3}, z_{k-2}\right)= \\
\left\{\begin{array}{c}
\frac{G\left(y_{k-1}-R_{\min }\right)-G\left(z_{k-3}\right)}{\left(G\left(z_{k-2}\right)-G\left(z_{k-3}\right)\right)\left(R_{\max }-R_{\min }\right)}, \\
z_{k-3}+R_{\min } \leq y_{k-1}<z_{k-3}+R_{\min } \\
\frac{G\left(y_{k-1}-R_{\min }\right)-G\left(y_{k-1}-R_{\max }\right)}{\left(G\left(z_{k-2}\right)-G\left(z_{k-3}\right)\right)\left(R_{\max }-R_{\min }\right)}, \\
z_{k-3}+R_{\max } \leq y_{k-1}<z_{k-2}+R_{\min } \\
G\left(z_{k-2}\right)-G\left(y_{k-1}-R_{\max }\right) \\
\frac{\left(G\left(z_{k-2}\right)-G\left(z_{k-3}\right)\right)\left(R_{\max }-R_{\min }\right)}{z_{k-2}+R_{\min } \leq y_{k-1}<z_{k-2}+R_{\max }}
\end{array}\right.
\end{gathered}
$$

Due to the fact that $z_{k-1}$ is decided by the largest endpoint location of the $(k-1)$-hop transmitters, the conditioned pdf of $h\left(z_{k-1} \mid z_{k-3}, z_{k-2}, m_{k-1}\right)$ can be calculated according to the order statistics, i.e.,

$$
\begin{array}{r}
h\left(z_{k-1} \mid z_{k-3}, z_{k-2}, m_{k-1}\right)=m_{k-1} \eta_{Y}\left(z_{k-1} \mid z_{k-3}, z_{k-2}\right) \\
{\left[H_{Y}\left(z_{k-1} \mid z_{k-3}, z_{k-2}\right)\right]^{m_{k-1}-1}}
\end{array}
$$

where the conditional cdf $H_{Y}\left(z_{k-1} \mid z_{k-3}, z_{k-2}\right)$ can be calculated from the conditional pdf $\eta_{Y}\left(z_{k-1} \mid z_{k-3}, z_{k-2}\right)$.

Eq. (10) to (16) allow us to determine the joint pdf of the endpoint locations and numbers of 0-hop, 1-hop, ...., $(k-1)$ hop transmitters conditioned on having $m$ relay nodes:

$$
\begin{gathered}
\xi\left(z_{k-1}, z_{k-2}, \ldots, z_{0}, N_{k-1}=m_{k-1}, \ldots, N_{1}=m_{1} \mid m\right) \\
=\frac{m !}{(m-k+1) !}\left(\begin{array}{c}
m-k+1 \\
q_{1}, q_{2} \ldots, q_{k-1}, q_{k}
\end{array}\right) \\
{\left[G_{X}\left(z_{k-2}\right)-G_{X}\left(z_{k-3}\right)\right] \ldots\left[G_{X}\left(z_{0}\right)-G_{X}(0)\right]} \\
\eta_{Y}\left(z_{k-1} \mid z_{k-3}, z_{k-2}\right) \ldots \eta_{Y}\left(z_{1} \mid 0, z_{0}\right) \\
{\left[H_{Y}\left(z_{k-1} \mid z_{k-3}, z_{k-2}\right)\left(G_{X}\left(z_{k-2}\right)-G_{X}\left(z_{k-3}\right)\right)\right]^{q_{k-1}} \ldots} \\
{\left[H_{Y}\left(z_{1} \mid 0, z_{0}\right)\left(G_{X}\left(z_{0}\right)-G_{X}(0)\right)\right]^{q_{1}}\left[G_{X}(L)-G_{X}\left(z_{k-2}\right)\right]^{q_{k}}}
\end{gathered}
$$

where $m=m_{1}+m_{2}+\ldots+m_{k}, q_{i}=m_{i}-1$ for $i=$ $1,2, \ldots, k-1$ and $q_{k}=m_{k}$. Then it can be derived $m-k+1=$ $q_{1}+q_{2}+\ldots+q_{k}$.

Therefore, (8) can be derived using (17) and multinomial theorem,

$$
\begin{gathered}
\operatorname{Pr}\left(z_{k-1} \geq L, z_{k-2}<L \mid m\right)= \\
\int_{R_{\min }}^{R_{\max }} \int_{z_{0}}^{L} \ldots \int_{z_{k-3}}^{L} \int_{L}^{z_{k-2}+R_{\max }} \frac{m !}{(m-k+1) !} \\
\prod_{k=2}^{K_{m}}\left[G_{X}\left(z_{k-2}\right)-G_{X}\left(z_{k-3}\right)\right] \eta_{Y}\left(z_{k-1} \mid z_{k-3}, z_{k-2}\right) \\
\left\{\sum_{k=2}^{K_{m}}\left[H_{Y}\left(z_{k-1} \mid z_{k-3}, z_{k-2}\right)\left(G_{X}\left(z_{k-2}\right)-G_{X}\left(z_{k-3}\right)\right)\right]\right. \\
\left.+\left[G_{X}(L)-G_{X}\left(z_{K_{m}-2}\right)\right]\right\}^{m-k+1} d z_{k-1} \ldots d z_{0}
\end{gathered}
$$

Then $P_{\text {suc }}(L)$ can be calculated by (18) together with (3).

Finally, the expected number of the forwarding nodes conditioned on having $m$ relay node can be expressed as

$$
\begin{gathered}
E[M(L) \mid m]=m P_{\text {suc }}(L \mid m)+\sum_{k=1}^{K_{m}}\{ \\
\sum_{m_{1}, m_{2}, \ldots, m_{k-1} \in \Gamma^{i=1}} \sum_{i}^{k-1} m_{i}\left[\operatorname { P r } \left(z_{k-1} \leq z_{k-2}<L,\right.\right. \\
\left.N_{k-1}=m_{k-1}, \ldots, N_{1}=m_{1} \mid m\right) \\
+\operatorname{Pr}\left(z_{k-2}<z_{k-1}<L, N_{k}=0, N_{k-1}=m_{k-1},\right. \\
\left.\left.\left.\ldots, N_{1}=m_{1} \mid m\right)\right]\right\}
\end{gathered}
$$

where $z_{k-1} \leq z_{k-2}$ implies that the endpoint location of the farthest $(k-1)$-hop transmitter is no larger than that of the farthest $(k-2)$-hop transmitter and the transmission fails; $z_{k-2}<z_{k-1}<L$ and $N_{k}=0$ implies that no $k$-hop transmitter exists despite $z_{k-2}<z_{k-1}$ and the transmission fails.

$$
\begin{gathered}
\operatorname{Pr}\left(z_{k-1} \leq z_{k-2}<L, N_{k-1}=m_{k-1}, \ldots, N_{1}=m_{1} \mid m\right) \\
=\int_{R_{\min }}^{R_{\max }} \int_{z_{0}}^{L} \ldots \int_{z_{k-3}}^{L} \int_{z_{k-3}}^{z_{k-2}} \\
\xi\left(z_{k-1}, \ldots, z_{0}, N_{k-1}=m_{k-1}, \ldots, N_{1}=m_{1}\right) \\
d z_{k-1} \ldots d z_{0}
\end{gathered}
$$

and $\operatorname{Pr}\left(z_{k-2}<z_{k-1}<L, N_{k}=0, N_{k-1}=m_{k-1}, \ldots, N_{1}=\right.$ $\left.m_{1} \mid m\right)$ can be calculated by the same method.

Then, $E[M(L)]$ can be calculated by (19) together with (3).

\section{Remark 1}

When $R_{\min }=R_{\max }=R$, the connection model degrades to an unit disk model. Therefore, the farthest $k$-hop transmitter is the node of the farthest location among all the $k$-hop transmitters under the unit disk model.

\section{Remark 2}

When $R_{\text {min }}=0$ and $G(x)$ is a linear function of $x$ (i.e. $G(x)=x / L)$, we can obtain $\operatorname{Pr}\left(z_{k-1} \geq L, z_{k-2}<L \mid m\right) \leqslant$ $\frac{n ! R_{\max }}{(n+k) !\left(R_{\max }-R_{\min }\right)^{k-1}} \sum_{q_{1}+q_{2}+\ldots+q_{k}=m-k+1} \prod_{j=1}^{k-1}\left(q_{j}+1\right)$ for $k \geqslant 2$ by 1) $\eta_{Y}\left(z_{k-1} \mid z_{k-3}, z_{k-2}\right) \leqslant \frac{1}{R_{\max }-R_{\min }}$ which can be derived from (14) and (15);2) the cdf $H_{Y}\left(z_{k-1}\right.$ $\left.z_{k-3}, z_{k-2}\right) \leqslant 1$ which is true eternally; 3) the normalization for $z_{i}$, i.e., $z_{i}^{\prime}=z_{i} / L$, which is the necessary condition for dirichlet integral.

\section{A. Design of Optimal Forwarding Probability Function}

As our analysis in Section III, the forwarding probability function $p(x)$ plays an important role in determining the 
performance of the forwarding scheme. In this subsection we analyze the design of the optimal forwarding probability function for the optimization problem:

$$
\begin{array}{lc}
\min _{p(x)} & E[M(L)] \\
\text { s.t. } & P_{\text {suc }}(L) \geq 1-\varepsilon
\end{array}
$$

The analytical expressions for $P_{\text {suc }}(L)$ and $E[M(L)]$ in their present form do not allow us to readily analyze the optimal functional form of $p(x)$ that solves the optimization problem. However, for particular forms of $p(x)$, e.g. $p(x)=c$ and $p(x)=a x$, the optimum parameters for $p(x)$ can be found using the method of Lagrange multipliers and solved numerically.

In the following analysis, we consider the simple case that $p(x)=c$ as an example. The optimization problem now reduces to finding the value of $c_{o p t}$ :

$$
\begin{array}{cc}
c_{\text {opt }}=\underset{p(x)=c}{\operatorname{argmin}} & E[M(L)] \\
\text { s.t. } & P_{\text {suc }}(L) \geq 1-\varepsilon
\end{array}
$$

The Lagrangian of the optimization problem can be written as

$$
\mathcal{L}(p(x)=c, \eta)=E[M(L)]+\eta\left(1-\varepsilon-P_{\text {suc }}(L)\right)
$$

The optimum value of $c$ can then be obtained by setting the partial derivative of $\mathcal{L}(p(x)=c, \eta)$ with regards to $c$ and with regards to $\eta$ to 0 respectively and solving the equations. Then we can derive that $E[M(L)]$ and $P_{\text {suc }}(L)$ are non-decreasing function of $c$. Based on the observation, it follows that the optimum value of $c$ is the solution to the equation $P_{\text {suc }}(L)=$ $1-\varepsilon$, which can be found numerically.

\section{Simulation}

In this section, we use simulations to establish the performance of the proposed uncoordinated cooperative forwarding scheme and provide some intuitively digestible results. Each point in the simulation is the average value obtained from 20000 random simulations. Considering a 1D axis, $n$ nodes between the source and the destination are deployed following a uniform distribution $U(0, L)$. The transmission ranges of all nodes follow a uniform distribution $U\left(R_{\min }, R_{\max }\right)$ where $R_{\min }=5 \mathrm{~m}$ and $R_{\max }=10 \mathrm{~m}$. Apart from the distribution of the node location and node transmission range, we found that the the number of nodes $n$, the distance between source and destination $L$ and the forwarding probability design affect the network performance according to the above analytical results. Therefore, we explore the effects of

- the number of nodes $n$ by assigning $n=10,30$ and 50. ;

- the distance between source and the destination $L$ varies from $10 \mathrm{~m}$ to $100 \mathrm{~m}$;

- the forwarding probability function design. We compares the performance of uncoordinated cooperative forwarding schemes using four different forwarding probability functions, i.e. $p(x)=c, p(x)=\frac{2 c x}{L}, p(x)=\frac{2 c}{R_{a}}\left(x-t R_{a}\right)$

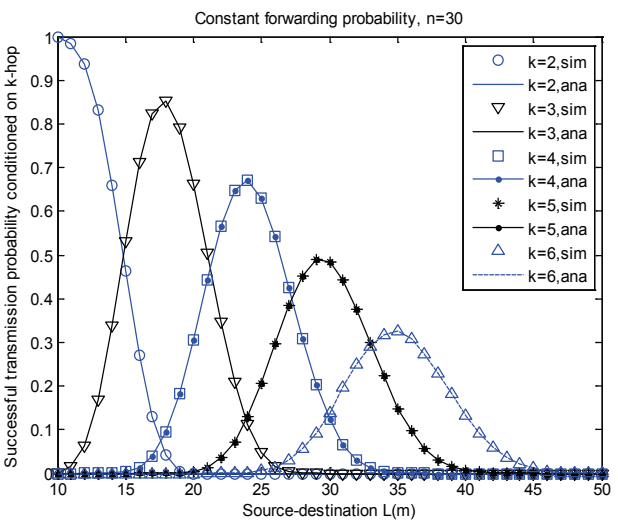

Fig. 2. The successful transmission probability on $k$-hop (i.e. $\operatorname{Pr}\left(z_{k-1} \geq\right.$ $\left.\left.L, z_{k-2}<L\right)\right)$

where $R_{a}$ is the period length and $t=\left\lfloor\frac{x}{R_{a}}\right\rfloor$, and $p(x)=\left\{\begin{array}{ll}0, & x \in\left(k R_{a},\left(k+\frac{1}{2}\right) R_{a}\right) \\ 2 c, & x \in\left[\left(k+\frac{1}{2}\right) R_{a},(k+1) R_{a}\right]\end{array}\right.$ where $k=$ $\left.0,1,2, \ldots \mid \frac{x}{R_{a}}\right\rfloor$. The design of functions guarantee that the number of relay nodes are same under the four forwarding probability functions, i.e. $\int_{0}^{L} p(x) d x=c L$. In our paper, we set $c=0.5$. In the following figures, the four cases are labeled as 'Constant', 'Linear', 'Periodically linear' and 'Periodically step' respectively.

on the successful transmission probability $P_{s u c}(L)$ and expected number of forwarding nodes $E[M(L)]$.

It is shown in Fig. 2 that the analytical result and the simulation result match well. The lines in Fig. 2 express that the probability that the destination to be reached by more hops increases with the growth of $L$. Due to the space limit, we omit the simulation results of other forwarding probability functions.

Since the design of functions guarantee that the number of relay nodes are same under the four forwarding probability functions, we only compare the successful transmission probability under the four forwarding functions. From Fig. 3, we can see that the increasing $n$ results in higher $P_{\text {suc }}(L)$ for all the four forwarding probability functions at the cost of more transmissions. Moreover, it is clear that the constant forwarding probability and the two periodical functions are of the similar performance, while the linear function performs poor. This provides an interesting result that the constant forwarding probability function is a potential one for the uncoordinated multi-path and multi-hop forwarding transmission. It is also shown in Fig. 4 that reducing the period of periodic function can further improve $P_{\text {suc }}(L)$ and an extreme case is the period approaches to 0 , i.e., the constant probability forwarding function. The result in Fig.4 further verifies that the constant forwarding probability function is the most potential one in uncoordinated multi-hop multi-path forwarding. 


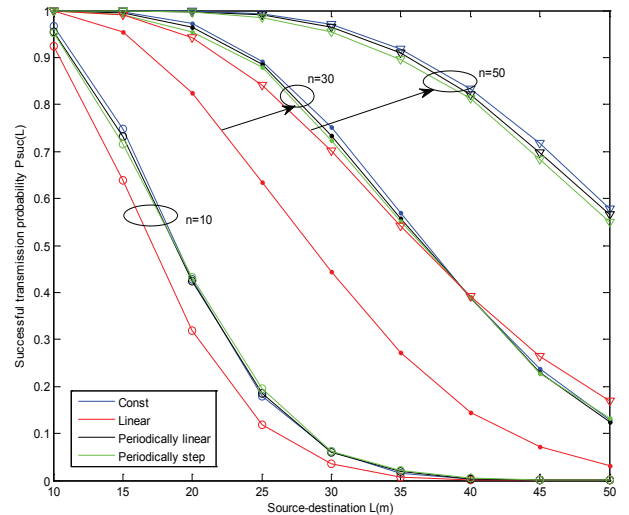

Fig. 3. $P_{\text {suc }}(L)$ on different number of relay nodes $n$ under four forwarding probability functions

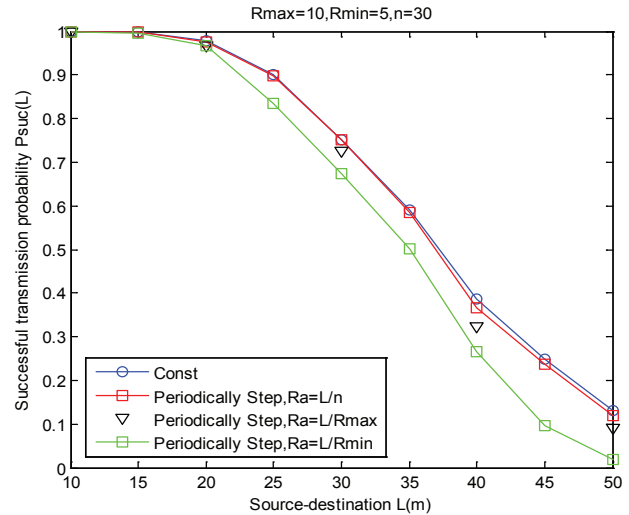

Fig. 4. $P_{\text {suc }}(L)$ on different period length

Fig. 5 compares the successful transmission probability using a linear function under the fixed transmission range and the random transmission range respectively. The fixed transmission range $R=E[r]=\frac{R_{\min }+R_{\max }}{2}$, which has same average value with the random transmission range. The network under the random transmission range has a higher probability of successful transmission probability than that under a fixed transmission range. The result verifies that the randomness of transmission range can improve $P_{\text {suc }}(L)$,

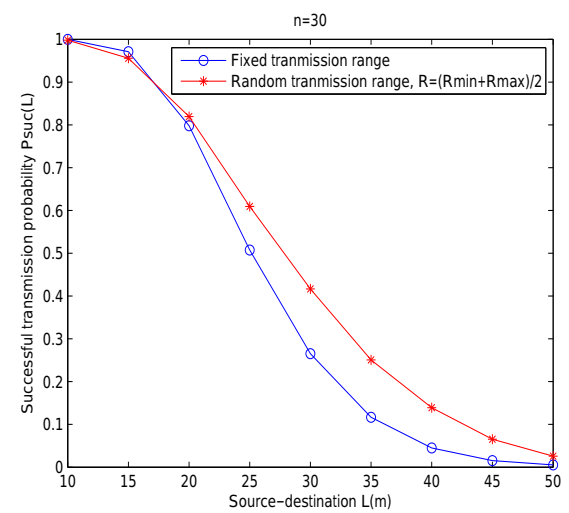

Fig. 5. $P_{\text {suc }}(L)$ on fixed and random transmission range which is consistent with our earlier work in [10].

\section{Conclusions}

This paper proposes an uncoordinated cooperative forwarding scheme, where each node with random transmission range. The exact analytical result on successful transmission probability and the expected number of forwarding nodes are derived considering the correlation between multi-path and multi-hop transmissions from the source to the destination. On this basis, an optimum uncoordinated cooperative forwarding probability function is proposed. The simulations and the corresponding discussions show that the constant forwarding probability function is the most potential one.

\section{ACKNOWLEDGEMENT}

This work is supported by the National Natural Science Foundation for Distinguished Young Scholar of China (Grant No. 61325006) and the Joint Research Fund for Overseas Chinese, Hong Kong and Macao Young Scientists of the National Natural Science Foundation of China (Grant No. 61428102)

\section{REFERENCES}

[1] S. Panichpapiboon and C. Lin, "Irresponsible forwarding under real intervehicle spacing distributions," IEEE Transactions on Vehicular Technology, vol. 62, no. 5, pp. 2264-2272, 2013.

[2] G. Mao, "Research on wireless multi-hop networks: Current state and challenges," Journal of Communications, Special Issue on Future Directions in Computing and Networking, vol. 7, no. 5, pp. 357-364, 2012.

[3] X. Lixiang, L. Libman, and M. Guoqiang, "Uncoordinated cooperative communications in highly dynamic wireless networks," IEEE Journal on Selected Areas in Communications, vol. 30, no. 2, pp. 280-288, 2012.

[4] Z. Chao, Z. Wei, and M. Guoqiang, "Uncoordinated cooperative communications with spatially random relays," IEEE Transactions on Wireless Communications, vol. 11, no. 9, pp. 3126-3135, 2012.

[5] S. Panichpapiboon and W. Pattara-Atikom, "A review of information dissemination protocols for vehicular ad hoc networks," IEEE Cоттиnications Surveys Tutorials, vol. 14, no. 3, pp. 784-798, 2012.

[6] S. Dulman, M. Rossi, P. Havinga, and M. Zorzi, "On the hop count statistics for randomly deployed wireless sensor networks," Int. J. Sen. Netw., vol. 1, no. 1/2, pp. 89-102, 2006.

[7] J. Andrews, S. Shakkottai, R. Heath, N. Jindal, M. Haenggi, R. Berry, G. Dongning, M. Neely, S. Weber, S. Jafar, and A. Yener, "Rethinking information theory for mobile ad hoc networks," IEEE Communications Magazine, vol. 46, no. 12, pp. 94-101, 2008.

[8] Z. J. Haas, J. Y. Halpern, and L. Li, "Gossip-based ad hoc routing," IEEE/ACM Transactions on Networking, vol. 14, no. 3, pp. 479-491, 2006.

[9] K. Jae-soo, Z. Qi, and D. P. Agrawal, "Probabilistic broadcasting based on coverage area and neighbor confirmation in mobile ad hoc networks," in IEEE Global Telecommunications Conference Workshops, 2004, pp. 96-101.

[10] Zijie Zhang, Guoqiang Mao and Brian Anderson, "Cooperative information forwarding in vehicular networks subject to channel randomness," IEEE ICC 2014, pp. 324-329, 2014.

[11] S. A. B. Wicker and S. B., "Connectivity of finite wireless networks with random communication range nodes," IEEE ICC 2009, pp. 1-5, 2009.

[12] A. F. S. Holst and Lars, "Covering the circle with random arcs of random sizes," Journal of Applied Probability, vol. 19, no. 2, pp. 373-381, 1982. 